\title{
Association Analysis of Candidate Quantitative Trait Loci for Resistance to Banded Leaf and Sheath Blight in Maize
}

\author{
Haijian Lin, Pengfei Leng, Guangtang Pan, and Zhiming Zhang
}

\begin{abstract}
Association mapping can be used for mapping and identifying genes control complex maize traits including diseases. In this study, the genetic structure of 198 inbred lines association population was conducted using 64 SSR markers by association mapping analysis, as well as the association for QTLs contribute resistance to banded leaf and sheath blight (BLSB). It could be referred to 6 subgroups including Lan, PA, PB, BSSS, SPT and LRC, and distributed uniformly. According to two years BLSB resistance identification, most of the inbred lines were high susceptible, few of them were resistance. 26 loci distributed in Chromosome 2, 3, 4, 5, 7, 8, 9, and 10 were discovered through the association mapping for resistance to BLSB using TASSEL, in which umc1202, umc2190 and umc1505 were significant related to the resistance $(p<0.05)$, while umc214 was significant in two years association mapping for relative RHDS (relative height of disease spot). Compared with the previous mapping results, more than half of the 26 loci in our research were reported in previous disease-resistance studies while part of them were consistent in BLSB resistance-related traits. Among these consensus loci, dupssr06, umc2164 and umc2287 were associated with disease index, bnlg666 and umc1858 were associated with the EH (ear height), which were consistent with our previous results of linkage analysis for QTL controlling BLSB.
\end{abstract}

Index Terms-Maize, banded leaf and sheath blight, QTL analysis, association mapping.

\section{INTRODUCTION}

Rhizoctonia solani Kühn is one of the most severe diseases happened worldwide maize belts. It was first reported by Voorhees that Rhizoctonia disease in maize ear caused by Rho-ctonis zeae occurred in southern of the US [1]. During the 1960s and 1970s, the continuous occurrence of R. solani Kühn was also reported in India, Japan, South Africa and Russia. In China, the first report of BLSB was in Jilin Province in 1966 [2]. This disease spread rapidly due to the increasing maize planting area and high-density cultivation, with the nationwide occurrence and increasing severity in present days. Grain yield decreased with rising positions of infected sheathes and worsening infection conditions of ears, which might be attributed to the reduction of weight per thousand kernels [3]. The morbidity was about $40 \%$ per year, and could reach $70 \%$ when it is serious (it could reach $100 \%$ in some region or some cultivar) [4]. The yield reduction

Manuscript received March 24, 2013; revised May 30, 2013. This work was supported in part by the National Hi-Tech program of China (2012AA10307).

The authors are with Key Laboratory of Biology and Genetic Improvement of maize in Southwest Region, Ministry of Agriculture; They are also with Maize Research Institute of Sichuan Agriculture University, Wenjiang, 611130, China (e-mail: linhj521@yahoo.com.cn). caused by BLSB ranged from $10 \%$ to $20 \%$, and can reach $35 \%$ when it was serious [5]. With the advent of global warming, the lost caused by BLSB tends to be worse.

Lots of reports showed that BLSB was a quantitative trait controlled by minor polygenes. By using the maize crossing combination CML270 $\times$ Ye478 backcross population and combined with IM and CIM methods, some QTLs were found related to BLSB resistance traits including relative resistance index, height of disease spot (HDS), and relative height of disease spot (RHDS), and two QTLs increase the relative resistance index or lowering the relative/absolute height were mapped in the interval of 102.64-113.61 cM in Chromosome 1 [6]. According to the result of Zhao et al. [7], $\mathrm{F}_{2}$ separation population of $\mathrm{R} 15 \times \mathrm{Ye} 478$ were constructed and used for constructing a linkage map with 146 SSR markers and an average interval of $11.4 \mathrm{cM}, 11$ resistance QTLs were detected in Chromosome 1, 2, 3, 4, 5, 6, 10, and 4 QTLs distributed in Chromosome 2, 6, 10 were detected in two testing locations. 4 resistance QTLs were detected in Chromosome 6, 7, 10 by using a population of $F_{2}$ of CML246 (resistant) $\times$ DM9 (susceptible), and two of them located in Chromosome 6 were linked to bnlg107 and umc1796 respectively, and $15.21 \%$ and $5.42 \%$ of the phenotype covariance could be explained by genetic effects respectively [8]. QTL mapping for BLSB resistance had been conducted widely in Triticum aestivum [9]-[11] and Oryza stalia [12], [13].

Most of the studies mentioned above were based on linkage genetic analysis which had shortcomings of long cycle length, requirement for high quality parents, and low mapping accuracy. Association mapping, based on linkage disequilibrium, greatly improved mapping effect and solution, had been widely used for gene mapping for complex traits in crops. 16 agronomy and quality traits of local soybean cultivars (393 representative materials) and wild populations (196 representative materials) was conducted by GWAS, 27 QTL related to traits in cultivated populations were identified [14]. Not only can association mapping complement the information of QTL linkage mapping, but also improve the breeding effect by providing allele variation information for parent selection, combination crossing and progency assisted selection. [15] glomerella resistance gene of lettuce was studied by association mapping and SNP marker Cntg10102 was significantly associated identified, and then the resistance materials could be isolated and applied to cultivar improvement. As for maize, Tharnberry [16] reported association mapping for flowering stage variations in 2001 ; association mapping for oil content [17], $\mathrm{PH}$ (plant height), smut resistance and water content of kernel [18] was conducted subsequently; Tian et al. (2010) identified genes 
related to the included angle of leaf and stem by GWAS. However, there was no report of analysis mapping for QTLs of BLSB resistance in maize [19].

\section{MATERIALS AND MethodS}

\section{A. Plant Materials and Phenotyping}

Association mapping population consisted of 198 tropic, semi-tropic and temperate maize inbred lines came from China, USA, and CYMMIT (see Table I). Population materials were bred in the same experiment spot of Maize Research Institute of Sichuan Agricultural University, and a randomized complete block designed with two replications and single row was applied for two years. Wheat seeds infected by $R$. solani AG1-IA were inoculated into the sheath nearest to the ground and kept closed; then water was sprayed to the plant base to keep humidity of the small environment in order to facilitate $R$. solani infection. HDS (Height of disease spot), EH (ear height), and PH (plant height) was measured at maturity stage according to the following standards:

TABLE I: INBRED LINES FOR TESTING

\begin{tabular}{|c|c|c|c|c|c|c|}
\hline Zong31 & Duo 212 & DH29 & CML326 & ES40 & B118 & Shen 135 \\
\hline Zhongzi03 & D863F & Duo16/AH & CML325 & EN46 & B107 & Qi319 \\
\hline Zhongzi 01 & D387 & Dong237 & CML317 & EN44 & B105 & MoP17 \\
\hline ZhongH204 & D375 & Dan599 & CML316 & EN40 & ANL6 & LXN \\
\hline Zhong69 & CMU3 & Dan 598 & CML306 & EN28 & ANL3 & $\begin{array}{l}\text { Liaogu00 } \\
1\end{array}$ \\
\hline Zhi41 & CML495 & Dan 360 & CML297 & EN25 & ANL11 & Liao785 \\
\hline Zheng36 & CML494 & Dan 340 & CML292 & EN12 & A 318 & Liao5114 \\
\hline Zheng 35 & CML493 & Dan 3130 & CML290 & 7327 & $975-12$ & Liao3180 \\
\hline Zheng 30 & CML489 & Chang7 & CML278 & 7319 & $835-2$ & Liao2345 \\
\hline Zheng 29 & CML487 & Chang7-2 & CML276 & 5311 & $698-3$ & Liao159 \\
\hline Zheng 28 & CML484 & Y731 & CML261 & 5213 & $286-4$ & Liao138 \\
\hline Zheng 22 & CML482 & y272 & CML256 & 4019 & $2002 F 34$ & Lian87 \\
\hline Yi1462 & CML480 & W138 & CML249 & 3411 & $2002 \mathrm{~F} 32$ & JH96C \\
\hline Ye478 & CML479 & V5 & CML192 & 3394 & $2002 \mathrm{~F} 30$ & JH59 \\
\hline Xi502 & CML473 & V4 & CML184 & 1058 & $18-599 \mathrm{R}$ & JF142 \\
\hline Tie9010 & CML472 & TZ18 & CML183 & 835 & $18-599 w$ & Ji853 \\
\hline Tie7922 & CML470 & TY30331-2 & CML182 & 501 & 526018 & Ji81162 \\
\hline Tian4 & CML457 & TY30331-1 & CML181 & 268 & 414259 & HTH \\
\hline Taixi113 & CML452 & STL6 & CML180 & 177 & 81565 & Hai9-21 \\
\hline $\mathrm{Si}-273$ & CML450 & STL5 & CML178 & 150 & 9642 & Shen 137 \\
\hline CA34514 & CA34501 & CA14707 & CA049Y01 & CA03116 & CA00308 & CA00106 \\
\hline CA34502 & CA3002 & CA14520 & CA03118 & CA00390 & CA00108 & C8605-2 \\
\hline C ML442 & CML435 & C ML426 & CML415 & CML398 & CML392 & CML385 \\
\hline C ML438 & CML429 & C ML423 & CML413 & CML396 & CML390 & CML383 \\
\hline C8605 & CML444 & C ML328 & CML348 & CML365 & CML376 & CML379 \\
\hline $\mathrm{Bt}$ & IRF291 & H95 & STL18 & STL17 & SA24 & S37 \\
\hline RP125 & R15 & R09 & P138 & O151 & NC250 & Mo17Ht \\
\hline STL20 & CML142 & K10 & KUI2007 & L102 & LX9801 & L 9801 \\
\hline B73 & B151 & & & & & \\
\hline
\end{tabular}


The 6 levels of disease severity were established: $0,1,3,5$, 7 , and 9, for which the RHDS was between $0,0.1$ to 0.25 , 0.25 to $0.5,0.5$ to $0.75,0.75$ to 1.0 and.1, respectively, leve 9 refer to the dead plant. The disease index (DI) was calculated according to the following formula: $\mathrm{DI}=\left[\sum\right.$ (severity class $\times$ plant numbers of this class)/ (the highest severity class $\times$ the total numbers of the investigated plants) $] \times 100$. And the resistance type were classified as follows: disease index of grown plants:0 - immune (I); 0-5.0: high resistance (HR); 5.1-10.1: resistance (R); 10.1-30.0: middle resistance (MR); 30.1-50.0: susceptible (S); 50.1-100: high susceptible (HS) [20]. Statistical analysis was done by Excel 2003.

\section{B. DNA Extraction and SSR Analysis}

\begin{tabular}{|c|c|c|c|c|c|}
\hline Name & Loci & Name & Loci & Name & Loci \\
\hline phi022 & 9.03 & umc1088 & 4.05 & dupssr06 & 9 \\
\hline umc1231 & 9.05 & $\begin{array}{l}\text { phi32817 } \\
5\end{array}$ & 7.04 & bnlg 1017 & 2.02 \\
\hline umc1069 & 8.08 & umc 2324 & 6.08 & umc1008 & 4.01 \\
\hline umc2322 & 6.06 & bnlg1031 & 8.06 & umc1019 & 5.06 \\
\hline bnlg2248 & 2.03 & bnlg 1525 & 9.07 & umc2027 & 4.05 \\
\hline $\mathrm{mmc} 0001$ & 3.09 & phi033 & 9.01 & bnlg666 & 8.05 \\
\hline dupssr-5 & 3 & umc1122 & 1.07 & umc1014 & 6.04 \\
\hline nc007 & 5.01 & umc1296 & 6.06 & bnlg 1538 & 6.01 \\
\hline bnlg1909 & 2.05 & $\begin{array}{l}\text { phi } 42070 \\
1\end{array}$ & 8 & umc1993 & $\begin{array}{l}10.0 \\
6\end{array}$ \\
\hline umc1143 & 6 & bnlg2235 & 8.02 & bnlg 1805 & 7.03 \\
\hline umc2214 & 2.1 & $\begin{array}{l}\text { phi19322 } \\
5\end{array}$ & 3 & $\begin{array}{l}\text { phi39616 } \\
0\end{array}$ & 5 \\
\hline bnlg161 & 6 & bnlg1940 & 2.08 & bnlg1338 & 2.01 \\
\hline phi065 & 9.03 & umc 2137 & $4.08-4.09$ & bnlg 2132 & 7 \\
\hline umc1202 & 8.03 & $\mathrm{mmc} 0022$ & 3.05 & umc2287 & 4.09 \\
\hline phi118 & 10 & bnlg 1178 & 1.02 & umc1080 & 2.06 \\
\hline umc1384 & 8.08 & umc 1858 & 8.04 & umc1154 & 7.05 \\
\hline phi021 & 4.03 & bnlg1443 & 6.05 & bnlg 1583 & 9.01 \\
\hline phi052 & $\begin{array}{l}10.0 \\
2\end{array}$ & umc 2190 & 7.06 & umc2136 & 5.08 \\
\hline bnlg108 & 2.04 & bnlg 2122 & 9.01 & phi054 & $\begin{array}{l}10.0 \\
3\end{array}$ \\
\hline dupssr12 & 1.08 & umc1789 & 9.06 & phi094 & 1.09 \\
\hline $\begin{array}{l}\text { phi10918 } \\
8\end{array}$ & 5.03 & bnlg 1258 & 2.08 & umc1505 & 9.07 \\
\hline bnlg1714 & 9.04 & bnlg1179 & 1.01 & bnlg 1808 & 7.02 \\
\hline bnlg1325 & 3.02 & umc2164 & 5.05 & & \\
\hline
\end{tabular}

DNA was extracted via $2 \times$ CTAB method [22], and its concentration and purity were measured by F-4500 spectrometer, then diluted to $20-40 \mathrm{ng} / \mu \mathrm{L}$. The polymorphic SSR primers distributed uniformly in chromosomes and were selected and shown in Table II. The PCR reaction system was $15 \mu \mathrm{l}$ and its components were as follows: $\mathrm{ddH}_{2} \mathrm{O} 10.2 \mu \mathrm{l}$, Taq-Buffer (10×Mg-free) $1.5 \mu \mathrm{l}, \mathrm{MgCL}_{2}\left(25 \mathrm{mmolL}^{-1}\right) 0.7 \mu \mathrm{l}$, dNTP-Mix (10 $\mathrm{mmolL}^{-1}$ each) $0.8 \mu \mathrm{l}$, Taq-Enzyme $\left(5 \mathrm{UH}^{-1}\right)$ $0.12 \mu \mathrm{l}$, Primers (F+R $1.0 \mu \mathrm{molL}^{-1}$ each) $0.4 \mu \mathrm{l}$, Template ( 30 ng $\left.\mu l^{-1}\right) 1 \mu 1$. Products were amplified by the landing-type PCR procedures, detailed procedures are showed in Table III. The quality of the amplification products were checked on $3 \%(\mathrm{w} / \mathrm{v})$ agarose gels.

TABLE III: PCR AMPLIFICATION PROCEDURES

\begin{tabular}{llllll}
\hline Step & Temperature & Time & Step & Tempreature & Time \\
& & & & & \\
\hline 1 & $94^{\circ} \mathrm{C}$ & $5 \mathrm{~min}$ & 7 & $94^{\circ} \mathrm{C}$ & $30 \mathrm{~s}$ \\
2 & $94^{\circ} \mathrm{C}$ & $40 \mathrm{~s}$ & 8 & $57^{\circ} \mathrm{C}$ & $30 \mathrm{~s}$ \\
3 & $66^{\circ} \mathrm{C}$ & $30 \mathrm{~s}$ & 9 & $72^{\circ} \mathrm{C}$ & $40 \mathrm{~s}$ \\
4 & -1 & Cycle & 10 & GO TO 7 & 30 times \\
5 & $72^{\circ} \mathrm{C}$ & $40 \mathrm{~s}$ & 11 & $72^{\circ} \mathrm{C}$ & 8 min \\
6 & GO TO 2 & 10 times & 12 & $12^{\circ} \mathrm{C}$ & preserving \\
\hline
\end{tabular}

\section{Data Anlaysis}

The software STRUCTURE was used for population structure analysis. The analyzing principle was: assuming type number of the allelic variation frequency characteristics in samples is $\mathrm{K}$ (subgroups obeying the Weinberger balance, where $\mathrm{K}$ is unknown), SSR loci of each subgroups were characterized by a set of allele variation frequencies, and materials in the sample were subsumed to (or estimated by Basyesian model) to the Kth subgroup, making loci frequencies within the subgroup follow the same Hardy-Weinberg equilibrium). Specific operating method in this experiment is as follows; First, $\mathrm{K}$ value was set range from 1 to 9 , then conducting 6 iterative computation, bum-in time and MCMC repeat number in each operation were 500,00 , the genetic relationship was mixed and frequency of allele was related. Association mapping for BLSB resistance was conducted by TASSEL using GML model and combined with the phenotype data with genetic similarity coefficient acquired by structure analysis.

\section{RESULTS}

\section{A. Structure Analysis}

Set the $\mathrm{K}$ value ranging from 1 to 9 , then conducted 6 iterative computation, bum-in time and MCMC repeat number in each operation were set as 500,00, the genetic relationship in the model setting was mixed and the frequency of allele is related. Based on the LnP (D) value, K was 6. As can be seen from Fig. 1, the LnP (D) increased along with the increase of $\mathrm{K}$, and

LnP (D) varies sharply among different $\mathrm{K}$ values before $\mathrm{K}$ reached 6; and the ongoing change of $\mathrm{LnP}$ (D) tent to slow down hand be stable when the $\mathrm{K}$ reached 6 . Combined with previous results, the subgroup number was determined to 6 , which were SPT (TangSipingtou and its derived lines), LRC (Lvda Red Cob and its derived lines), Lancaster, BSSS (including reid), PA, PB, respectively. In general, all materials in this study were evenly distributed among six groups; BSSS (including Reid) had maximum number of materials which account for $21 \%$ of the total materials; and the rest 5 accounted for approximately $15 \%$ each (see Fig. 2). 
These results indicated that the abundant genetic structure of the mapping population was suitable for association analysis.

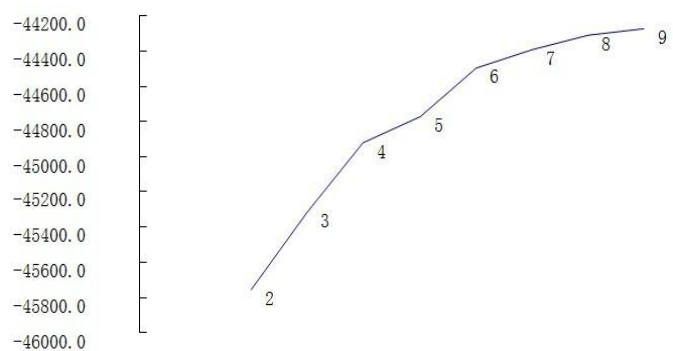

Fig. 1. Changing of K value.

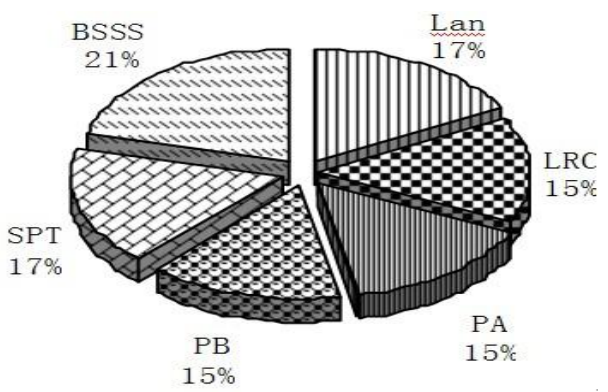

Fig. 2. Group distribution of materials.

B. Association Mapping for BLSB Resistance in Maize

A series of significant loci with $p$ value $<0.05$ were detected in five traits HDS, EH, RHDS, PH and DI (disease index) by the association mapping for investigation results of BLSB resistance traits in 2008 and 2009, detailed information could be seen in Table IV. In 2008, loci were significant in multiple traits including phi021 in HDS, EH and DI, phi328175 in the HDS, EH, RHDS, PH and DI, phi033 in HDS, EH and PH, bnlg2235 in HDS, EH and PH, umc1858 in HDS and EH, umc1858 in HDS and EH, umc2190 in HDS and PH, umc1789 in HDS, EH, RHDS and DI, bnlg 666 in all the five traits. In 2009, few loci were detected and only 3 of them were significant in two traits relatively, they were bnlg1909 in HDS and PH, umc2287 in $\mathrm{EH}$ and RHDS, phi054 in PH and DI. By integrating the loci detected in the two years, umc2164 was significant in RHDS in two years; and phi 033 was significant in RHDS exclusively in 2009, while it was not significant in 2008 but significant in HDS, $\mathrm{EH}$ and $\mathrm{PH}$ in 2008. From the prospective of variation explanation, the maximum value was in 2009 in HDS where the value of phi328175 reached 0.58, while the maximum value was in HDS in 2009 where the value of dupssr06 reached 0.2853 . The average value of explanation for trait variation in 2008 and 2009 respectively was 0.30 and 0.23 for HDS, 0.30 and 0.17 for $\mathrm{EH}, 0.30$ and 0.12 for RHDS, 0.34 and 0.20 for PH, 0.27 and 0.14 for DI.

TABLE IV: RESUltS OF ASSOCIATION MAPPING FOR MAIZE RESISTANCE TO BLSB IN 2008 AND 2009

\begin{tabular}{|c|c|c|c|c|c|c|c|}
\hline 2008 & Marker & p_Marker & Rsq_Marker & 2009 & Marker & p_Marker & Rsq_Marker \\
\hline \multirow[t]{9}{*}{ HDS } & $\begin{array}{l}\text { phi021 } \\
\text { phi32817 }\end{array}$ & 0.0469 & 0.1298 & HDS & $\begin{array}{l}\text { bnlg190 } \\
9\end{array}$ & 0.0367 & 0.177 \\
\hline & 5 & $6.78 \mathrm{E}-06$ & 0.58 & & dupssr06 & 0.002 & 0.2853 \\
\hline & phi033 & 0.0253 & 0.1094 & & & & \\
\hline & bnlg2235 & 0.0034 & 0.2912 & & & & \\
\hline & umc1858 & 0.0401 & 0.0831 & & & & \\
\hline & umc2190 & 0.015 & 0.1631 & & & & \\
\hline & umc1789 & $5.34 \mathrm{E}-05$ & 0.4802 & & & & \\
\hline & bnlg666 & $1.2 \mathrm{E}-08$ & 0.4746 & & & & \\
\hline & bnlg 1805 & $2.16 \mathrm{E}-06$ & 0.4002 & & & & \\
\hline \multirow[t]{9}{*}{ EH (Ear Height) } & $\begin{array}{l}\text { phi021 } \\
\text { phi32817 }\end{array}$ & 0.0493 & 0.1361 & EH (Ear Height) & dupssr-5 & 0.0466 & 0.2177 \\
\hline & 5 & $2.16 \mathrm{E}-05$ & 0.5625 & & nc007 & 0.024 & 0.1482 \\
\hline & phi033 & 0.0247 & 0.1099 & & $\begin{array}{l}\text { umc1384 } \\
\text { bnlg } 103\end{array}$ & 0.005 & 0.1388 \\
\hline & bnlg2235 & 0.0077 & 0.2723 & & 1 & 0.0407 & 0.2542 \\
\hline & umc1858 & 0.0442 & 0.0812 & & umc2287 & 0.011 & 0.0822 \\
\hline & umc2190 & 0.021 & 0.1552 & & & & \\
\hline & umc1789 & $1.08 \mathrm{E}-04$ & 0.4678 & & & & \\
\hline & bnlg666 & 8.98E-09 & 0.4783 & & & & \\
\hline & $\begin{array}{l}\text { bnlg } 1805 \\
\text { phi32817 }\end{array}$ & $2.03 \mathrm{E}-06$ & 0.4025 & & & & \\
\hline RHDS & 5 & 0.0424 & 0.4082 & RHDS & phi052 & 0.0253 & 0.1221 \\
\hline
\end{tabular}




\begin{tabular}{|c|c|c|c|c|c|c|c|}
\hline & bnlg 1525 & 0.0357 & 0.1597 & & phi033 & 0.0347 & 0.0999 \\
\hline & phi42070 & & & & bnlg 125 & & \\
\hline & 1 & 0.0143 & 0.312 & & 8 & 0.0337 & 0.0629 \\
\hline & umc1789 & 0.0238 & 0.356 & & umc2287 & 0.0134 & 0.0792 \\
\hline & bnlg 1017 & 0.0226 & 0.1269 & & umc2164 & 0.0356 & 0.2257 \\
\hline & bnlg666 & $1.47 \mathrm{E}-05$ & 0.3802 & & & & \\
\hline & bnlg 1805 & $8.35 \mathrm{E}-04$ & 0.2998 & & & & \\
\hline & umc2164 & $6.31 \mathrm{E}-04$ & 0.3373 & & & & \\
\hline & phi32817 & & & & bnlg190 & & \\
\hline $\mathrm{PH}$ & 5 & $8.44 \mathrm{E}-05$ & 0.5357 & $\mathrm{PH}$ & 9 & 0.0448 & 0.1721 \\
\hline & phi033 & 0.0238 & 0.1105 & & $\begin{array}{l}\text { phi054 } \\
\text { bnlo133 }\end{array}$ & 0.0081 & 0.2557 \\
\hline & bnlg2235 & 0.0084 & 0.2681 & & 8 & 0.0395 & 0.1566 \\
\hline & umc 2190 & 0.018 & 0.1576 & & & & \\
\hline & umc1789 & $1.41 \mathrm{E}-04$ & 0.4595 & & & & \\
\hline & bnlg666 & $5.95 \mathrm{E}-08$ & 0.4501 & & & & \\
\hline & bnlg1805 & 4.73E-06 & 0.387 & & & & \\
\hline DI & phi021 & 0.0471 & 0.1217 & DI & phi052 & 0.0457 & 0.1069 \\
\hline & $\begin{array}{l}\text { phi32817 } \\
5\end{array}$ & 0.0375 & 0.4117 & & umc 2287 & 0.0067 & 0.0903 \\
\hline & bnlg 1525 & 0.0431 & 0.1545 & & phi054 & 0.0154 & 0.2272 \\
\hline & phi42070 & & & & & & \\
\hline & 1 & 0.0101 & 0.3208 & & & & \\
\hline & bnlg2235 & 0.0343 & 0.2383 & & & & \\
\hline & umc1789 & 0.0173 & 0.3647 & & & & \\
\hline & bnlg1017 & 0.0346 & 0.1171 & & & & \\
\hline & bnlg666 & $1.58 \mathrm{E}-05$ & 0.378 & & & & \\
\hline & bnlg1805 & 0.001 & 0.2953 & & & & \\
\hline & umc2164 & 7.97E-04 & 0.332 & & & & \\
\hline
\end{tabular}

Abbreviates: DI=Disease Index; PH=Plant Height; BLSB=Banded Leaf Sheath Blight; HDS=Height of Disease Spot; RDHS= Relative Height of Disease Spot; MRDV= Maize Rough Dwarf Virus; EH=Ear Height.

An association mapping was conducted after the identification of resistance levels by integrating two years' resistance identifications. 3 associated loci including umc1202, umc2190 and umc1505, were detected significant at $p<0.05$ level; most variations among these loci could be explained by umc 2190 . In addition, umc 2190 was significant in the HDS, EH and PH, which corresponded to the results of loci detected in the traits.

\section{Discussion}

In this research, half of the 26 loci were reported previously, and some of them were reported related with BLSB resistance traits, and detailed information could be seen in Table V. Dupssr06 in DI, bnlg666 in EH, umc1858 in EH, umc2164 in DI and umc2287 in DI, are accordance with our previous work on BLSB QTL mapping. Some loci were involved in resistance to other diseases; such as umc1202 and bnlg2235 were involved in resistance to exserohilum turcicum in maize [22], especially that umc 1202 was directly involved in the closelinkage of exserohilum turcicum resistance gene Ht2 [23]; and further study could be conducted to investigate the $\mathrm{Ht} 2$ involvement in the formation of BLSB resistance; umc1505 involved in the formation of resistance to Maize Rough-Dwarf Virus Disease[24]; phi328175 might involve in the formation of resistance to Maize Silk Cut [25]; bnlg1258 involved in resistance to maize gibberellins [26]. These loci were detected in the BLSB resistance research, which may be attributed to the theory that they affect maize resistance to different disease via the same regulation mechanism such as the Programmed Cell Death and cell-wall thickening, and their specific was of involvements in the maize resistance can be further identified by physiology and biochemistry researches. The particular cases are as follows: the previous studies indicated that bnlg1909 involved in maize 
photosynthesis via regulating the synthesis of chlorophyll a and b [27]; bnlg1017 involved in construction of maize plant type by affecting leaf angle, leaf direction, plant height and height of ear [28], [29]; bnlg1525 involved in drought tolerance [30]. The possible reason for their involvements in BLSB resistance might be as follows: bnlg1909 improved the efficiency of photosynthesis to provide energy for disease resistance [27]; bnlg1017 reduced the infection probability via regulating the angle to make it difficult for germs to invade sheath[31], this may be attributed to the phytopathology theory that BLSB germ infect the plant by invading the sheath initially, the it elevated the ear height to reduce the possibility of ear infection to reduce the effect on maize quality; bnlg1525 involved in the abiotic stress [25], the mechanism may be that according to the physiology and biochemistry researches for drought tolerance, osmotic pressure adjustments, including cell-wall thickening, were involved in the drought tolerance, and the BLSB infection also causes dehydration and degreasing, thus those loci involved in drought tolerance may get involved in maize resistance to BLSB via these methods.

TABLE V: QTL of ShEATH BLight Disease RESistance COMPARED WITH THE PREVIOUS STUDIES

\begin{tabular}{|c|c|c|c|}
\hline Loci & Position & Association results of this study & Putative traits in Previous results \\
\hline phi054 & 10.03 & DI and PH in 2009 & $\begin{array}{l}\text { DI for BLSB } \\
\text { grey speck disease, Rust }\end{array}$ \\
\hline phi052 & 10.02 & in 2009 RHDS 、 DI & Not mentioned \\
\hline bnlg 1525 & 9.07 & in 2008 RHDS 、 DI & $\begin{array}{l}\text { Drought Tolerant } \\
\text { Grain Yield }\end{array}$ \\
\hline umc1505 & 9.07 & Integrated resistance level & Maize MRDV \\
\hline umc1789 & 9.06 & in 2008 HDS,EH,RHDS,PH,DI & Not mentioned \\
\hline phi033 & 9.01 & $\begin{array}{l}\text { in } 2008 \mathrm{HDS} 、 \mathrm{EH} 、 \mathrm{PH} \\
\text { in } 2009 \mathrm{RHDS}\end{array}$ & Not mentioned \\
\hline dupssr06 & 9 & in 2009 HDS & BLSB DI \\
\hline umc1384 & 8.08 & in $2009 \mathrm{EH}$ & Not mentioned \\
\hline bnlg1031 & 8.06 & in $2009 \mathrm{EH}$ & Not mentioned \\
\hline bnlg666 & 8.05 & $\begin{array}{l}\text { in } 2008 \mathrm{HDS} 、 \mathrm{EH} 、 \mathrm{RHDS} 、 \\
\mathrm{PH} \cdot \mathrm{DI}\end{array}$ & $\mathrm{EH}$ \\
\hline umc1858 & 8.04 & in $2008 \mathrm{HDS} \cdot \mathrm{EH}$ & $\mathrm{EH}$ \\
\hline umc1202 & 8.03 & Integrated resistance level & BLSB gene $H t 2$ close linkage \\
\hline bnlg2235 & 8.02 & $\begin{array}{l}\text { in } 2008 \mathrm{HDS} 、 \mathrm{EH} 、 \\
\text { RHDS 、DI }\end{array}$ & Maize BLSB \\
\hline phi420701 & 8 & in 2008 RHDS 、DI & Not mentioned \\
\hline umc 2190 & 7.06 & $\begin{array}{l}\text { in } 2008 \mathrm{HDS} 、 \mathrm{EH} \text { 、 } \\
\mathrm{PH} 、 \text { Integrated resistance level }\end{array}$ & $\mathrm{PH}$ \\
\hline phi328175 & 7.04 & $\begin{array}{l}\text { in } 2008 \mathrm{HDS} 、 \mathrm{EH} 、 \\
\text { RHDS } 、 \mathrm{PH} 、 \mathrm{DI}\end{array}$ & Maize silk cut \\
\hline bnlg 1805 & 7.03 & $\begin{array}{l}\text { in } 2008 \mathrm{HDS} 、 \mathrm{EH} 、 \\
\text { RHDS } 、 \mathrm{PH} 、 \mathrm{DI}\end{array}$ & Not mentioned \\
\hline umc2164 & 5.05 & $\begin{array}{l}\text { in } 2008 \text { RHDS - DI } \\
\text { in } 2009 \text { RHDS }\end{array}$ & BLSB DI \\
\hline nc007 & 5.01 & in $2009 \mathrm{EH}$ & Not mentioned \\
\hline umc 2287 & 4.09 & in $2009 \mathrm{EH} 、 \mathrm{RHDS} 、 \mathrm{DI}$ & BLSB DI \\
\hline phi021 & 4.03 & in $2008 \mathrm{HDS} \cdot \mathrm{EH} \cdot \mathrm{DI}$ & Not mentioned \\
\hline dupssr-5 & 3 & in $2009 \mathrm{EH}$ & Not mentioned \\
\hline bnlg 1258 & 2.08 & in 2009 RHDS & Corn Fusarium head blight \\
\hline bnlg 1909 & 2.05 & in $2009 \mathrm{HDS} \cdot \mathrm{PH}$ & Chlorophy a,b \\
\hline bnlg1017 & 2.02 & in 2008 RHDS 、 DI & $\begin{array}{l}\text { Angle and leaf direction } \\
\mathrm{PH} \cdot \mathrm{EH}\end{array}$ \\
\hline bnlg 1338 & 2.01 & in $2009 \mathrm{PH}$ & Not mentioned \\
\hline
\end{tabular}

Abbreviates: DI=Disease Index; PH=Plant Height; BLSB=Banded Leaf Sheath Blight; HDS=Height of Disease Spot; RDHS= Relative Height of Disease Spot; MRDV= Maize Rough Dwarf Virus; EH=Ear Height.

From the prospect of loci distribution in chromosomes, loci were detected in all the chromosomes except 
Chromosome1 and 6. A relatively large number of 7 loci were detected in Chromosome 8; two loci, including bnglg1525 and umc1505, were detected in Chromosome 9.07; thus fine mapping for these QTLs, such as employing candidate gene strategy and increasing marker density, could be used to confirm whether there is key QTL in this loci. Detected loci may not be associated with only one trait usually, and the associations among traits can be discussed using date of this study. Among these traits, HDS and EH coexist usually, as well as DI and RHDS; thus it can be confirmed that there must be some associations between these traits.

\section{REFERENCES}

[1] P. K. Voorhee, "Sclerotial rot of corn caused by Rhizoctonia zeae n.sp," Phytopath., vol. 24,pp.1209-1303,1934,2008

[2] P. K. Qi, J. K. Bai, and G. X. Zhu, Cultivated plant fungal disease history of Jilin province, Science Press, 1996.

[3] J. M. Yan et al., "Damage and yield loss of corn sheath blight," Maize Science., vo.16, no. 5, pp. 123-125, 2008.

[4] H. T. Tang, T. Z. Rong, and J. P. Yang, "Research progress of sheath blight in maize," Maize Science., vol. 12, no. 1, pp.93-96, 2004.

[5] J. Chen, Diagnosis and treatment of diseases of maize, Golden Shield Press., vol. 46, 1999.

[6] H. Yang et al., "Maize sheath blight resistance QTL molecular markers," Chinese Science Bulletin., vol. 50, no. 8, pp. 772-776, 2004.

[7] M. J. Zhao et al., "Quantitative trait loci mapping of resistan-ce genes to banded leaf and sheath blight in maize," Crop Science., vol. 46, pp. 1039-1045, 2006.

[8] G. P. Chen et al., "Resistance QTL maize sheath blight," Southwest China Journal of Agricultural Sciences., vol. 22, no. 4, pp. 950-955, 2009.

[9] X. C. Zhang et al., "Molecular marker analysis of QTL and the resistant gene of resistance to wheat sharp eyespot," Journal of plant genetic resources., vol. 6, no. 3, pp276-279, 2005.

[10] Y. Y. Tang et al., "QTL study of AR-Z wheat sheath blight resistance," Journal of Triticeae Crops., vol. 24, no. 4, pp. 11-16, 2004.

[11] L. J. Ren, S. B. Cai, and J. Z. Wu, "Study on SSR marker QTL resistance to sheath blight of wheat," Journal of Yangzhou University (agriculture and Life Science Edition), vol. 25, no. 4, pp. 11-16, 2004.

[12] K. Yasufumi, Q. Qian, S. Hiroyuki, S.Teng, D. L. Zeng, K. Fujimoto, and L.H. Zhu, "QTL Analysis of Sheath Blight Resistance in Rice(Oryza sativa L.)," Acta Genetica Sinica, vol. 29, no. 1, pp. 50-55, 2002.

[13] C. X Tan, X. M. Ji, Y. Yang, X. Y. Pan, S. M. Zuo, Y. F. Zhang, J. H. Zou, Z. X. Chen, L. H. Zhu, and X. B. Pan, "Identification and marker-assisted selection of two major quantitative genes controlling rice sheath blight resistance in backcross generations," Acta Genetica Sinica, vol. 32, no. 4, pp. 400-405, 2005.

[14] Z. X. Wen, T. J Zhao, Y. Z. Zheng, S. H. Liu, C. E. Wang, F. Wang, and J. Y. Gai, "Association analysis of agronomic and quality traits with SSR markers in glycine max and glycine soja in China: I. population structure and associated markers," Acta Agronomica Sinica, vol. 34, no. 7, pp. 1169-1178, 2008.

[15] I. Simko et al., "Association mapping and marker-assisted selection of the lettuce dieback resistance gege Tvrl," BMC Plant Biology, vol. 9, pp. 135, 2009.
[16] J. M. Thornsberry et al., "Dwarf8 polymor-phisms associate with variation in flowering time," Nature Genetics, vol. 28, pp. 286-289, 2001.

[17] C. C. Laurie et al., "The genetic architecture of response to long- term artificial selection for oil concentration in the maize kernel," Genetics, vol. 168, pp. 2141- 2155, 2004

[18] B. Parisseaux and R. Bemardo, "In silico mapping of quantitative trait loci in maize," Theor. Appl. Genet, vol. 109, pp. 508- 514, 2004.

[19] F. Tian et al., "Genome-wide association study of leaf architecture in the maize nested association mapping population," Nat Genet, doi:10.1038/ng.746,2010.

[20] C. E. Harjes et al., "Natural genetic variation in lucopene epsilon cyclase tapped for maize biofortification," Science, vol. 319, no. 5861, pp. 319-330, 2008.

[21] M. A. Saghai-Maroof et al., "Ribosomal DNAspacer length polymorphisms in barley:Mendelian inheritance, chromosomal location and population dynamics," in Proc. Natl. Acad. Sci. (USA), vol. 81, pp. 8014-8018, 1984.

[22] X. Y. Yin et al., "Fine mapping of corn leaf blight resistance gene Ht2," Chinese Science Bulletin, vol. 42, no. 23, pp. 1811-1814, 2002.

[23] X. J. Yang, M. Lu, S. H. Zhang, F. Zhou, Y. Y. Qu, and C. X. Xie, "QTL mapping of plant height and ear position in maize(Zea mays L.)," Hereditas, vol. 30, no. 11, pp. 1477-1486, 2008.

[24] Y. P. Chen, Q. C. Meng, and J. H. Yuan, "Identification of the molecular markers linked to the MRDV-resistance locus in maize using SSR-BSA technique," Jiangsu Journal of Agricultural Sciences, vol. 24, no. 5, pp.590-594, 2008.

[25] Y.X. Wei et al., "QTL mapping of resistance to silk cut in maize," Acta Sinica, vol.35, no. 4, pp. 741-744, 2009.

[26] S. G. Gordon, Genetic mapping and components of resistance to cercospora zeae-maydis in maize, The Ohio State University, 2003

[27] J. Leipner and E. Mayer, "QTL mapping in maize seedings reveals little relevance of $\mathrm{C} 4$ cycle enzymesand antioxidants for genotypic differences in chilling tolerance ofphotosynthesis," Maydica, vol. 53, pp. 269-277, 2008.

[28] M. Lu et al., "Construction of a SSR linkage map and mapping of quantitative trait loci (QTL) for leaf angle and leaf orientation with an elite maize hybrid," Hereditas, vol. 29, no. 9, pp. 1131-1138, 2007.

[29] X. Wei et al., "QTL mapping of resistance to silk cut in maize," Acta Sinica, vol. 35, no. 4, pp. 741-744, 2009.

[30] N. Xiao et al., "Quantitative trait locus analysis of drought tolerance and yieldin maize in China," Plant Mol Biol Rep, vol. 23, pp. 155-165, 2005.

[31] M. J. Zhao et al., "Initial identification of quantitative trait loci controlling resistance to banded leaf and sheath blight at elongating and heading date in maize," Journal of Molecular Cell biology, vol. 39, no. 2, pp. 139-144, 2006.

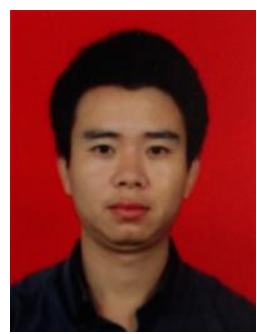

Haijian Lin was born on 28 May 1981 in Sichuan Province, China. He got his undergraduate degree in 2004, B.S.Mgt.Sci in Sichuan Agricultural University, China. He got his $\mathrm{PhD}$ in 2010 in Sichuan Agricultural University, China. His major is Plant Breeding and the specialization fields are plant disease resistance and haploid breeding. He is a regular research assistant at Sichuan Agricultural University, China from 2010.7 to now. 